\title{
Restricción calórica, ¿un camino para la prevención y tratamiento de la diabetes tipo 2?
}

\author{
Caloric restriction, a way for prevention and \\ treatment of type 2 diabetes?
}

\begin{abstract}
Caloric restriction is the only nutritional intervention that has shown solid benefits in health and longevity in experimental animals. On the positive side, those related to glucose homeostasis are striking and consistent. It seems that these benefits are associated with lower generation of mitochondrial reactive oxygen species, with less lipid peroxidation of cell membrane and stimulation of macroautophagy. Well known patterns such as the Mediterranean diet, vegan and vegetarian diet, the diet of the older population of Okinawa and the DASH (Dietary Approaches to Stop Hypertension) diet with a special attention to the unsaturated oils and strict respect for fasting between meals, might make an interesting line of construction of a dietary pattern aimed to prevention and treatment of type 2 diabetes.

Key words: Calorie restriction; diabetes; dietary patterns; treatment; prevention.
\end{abstract}

\section{INTRODUCCIÓN}

En los últimos años la investigación que relaciona la restricción calórica con la longevidad ha tomado fuerza. Estudios en insectos y mamíferos han demostrado que una dieta que restringe solo calorías, manteniendo un adecuado aporte de nutrientes esenciales logra aumentar los años de vida y retrasa la presencia de enfermedades (1-3).

Esta revisión, tiene el propósito de actualizar y analizar la abundante literatura existente respecto de este modelo de alimentación y su relación con una de las patologías con mayor prevalencia creciente y de gran impacto en los sistemas de salud de prácticamente todos los países, la diabetes tipo 2 (40).

Los experimentos en animales de laboratorio aportan pruebas abrumadoras del impacto que estas dietas tienen sobre la longevidad y la salud, los que se han desarrollado en condiciones de control, situación que ha permitido que el sujeto de la experiencia no tenga más opciones de alimentación que no sea la del protocolo de estudio. El ser humano, sin embargo, se enfrenta a un ambiente de permanente oferta alimentaria y de fácil acceso, condiciones que contribuyen como una de las causas de las enfermedades no trasmisibles.

\section{LA RESTRICCIÓN CALÓRICA (RC)}

Los estudios relacionados con la restricción calórica se inician con las observaciones en ratas, publicadas en 1935 por Mc Cay y col (1). Los investigadores desarrollaron un experimento en ratas sometidas a una dieta hipocalórica que satisfacía todas sus necesidades nutricionales. Descubrieron que aumentaba en una tercera parte la esperanza de vida media de esos animales.
Rafael Jiménez $L$.

Unidad Académica de la Carrera de Nutrición y Dietética, Departamento de Nutrición y Alimentos, Facultad de Farmacia, Universidad de Valparaíso, Valparaíso, Chile.

Dirigir la correspondencia a: Profesor Rafael Jiménez Lira Unidad Académica de la Carrera de Nutrición y Dietética Departamento de Nutrición y Alimentos Facultad de Farmacia Universidad de Valparaíso - Valparaíso Teléfonos: (032) 2508141 - 9-3349212 E-mail: rafael.jimenez@uv.c

Este trabajo fue recibido el 6 de Diciembre de 2011 y aceptado para ser publicado el 1 de Junio de 2012.

Al mismo tiempo, era menor el impacto de las enfermedades propias del senescente en comparación con el grupo control de ratas que seguían una dieta estándar. Experimentos posteriores corroboraron los efectos de RC sobre las enfermedades relacionadas con la edad (2 - 4). El fundamento central de la restricción calórica corresponde a lo que en inglés se expresa como "undernutrition without malnutrition" (reducción de la ingesta de calorías sin detrimento de la nutrición), lo que significa entregar todos los nutrientes de acuerdo a las necesidades del individuo, afectando sólo la ingesta energética. Monos sometidos a RC consumen entre 20\% y 50\% menos de calorías que los controles y, al mismo tiempo, reciben las proteínas, grasas, vitaminas y minerales que exige el funcionamiento eficaz de su organismo (5). Corresponde a regímenes diseñados para mantener un estado nutricional adecuado con modesta disminución calórica respecto de una alimentación ad libitum. Los animales con restricción calórica, son casi siempre más activos y sanos que su contraparte de alimentación ad libitum, que tienden a desarrollar obesidad (6).

De la totalidad de indicadores mensurables del envejecimiento, se estima que el $90 \%$ aproximadamente muestra características de mayor juventud por más tiempo, la respuesta inmunitaria se mantiene inalterable hasta una edad mucho más avanzada, del mismo modo, se observa una mayor sensibilidad a la insulina en los ratones sometidos a RC que sus pares controles (7). Si bien la mayor parte de los trabajos realizados con restricción calórica ponen en evidencia estos importantes hallazgos, aún se desconoce los mecanismos biológicos involucrados y, del mismo modo, su efecto en el ser humano. 


\section{COMO SE EXPLICAN LOS EFECTOS DE LA RESTRICCIÓN CALÓRICA}

Los resultados obtenidos en la investigación de la RC en modelos animales, han sido asombrosos, sin embargo, no existe consenso respecto de los mecanismos involucrados. Una de las teorías sostiene que la restricción calórica reduce la tasa de división celular en muchos tejidos, lo que explicaría en parte los efectos positivos de la RC sobre el cáncer (9). Otra teoría busca una explicación en la reducción del nivel de glucosa plasmático que provocaría la RC, lo que disminuiría la tasa de glicosilación de proteínas de larga duración (10). Sin embargo, la hipótesis que logra mayor consenso es la que postula que la restricción calórica lentifica el envejecimiento debido a que disminuye la producción de radicales libres o especies reactivas de oxígeno (ROS), señalados como los principales responsables del envejecimiento orgánico (8). Estas sustancias atacan continuamente a todos los tipos de macromoléculas biológicas, pudiendo aumentar significativamente en presencia de enfermedades. En el individuo sano hay una producción celular baja pero permanente de ROS. Mayoritariamente la producción de estas especies reactivas de oxígeno o radicales libres se origina en la cadena respiratoria mitocondrial. La afirmación de que los ROS de origen mitocondrial constituyen la principal causa del envejecimiento (11) incrementa cada vez más sus adherentes en la literatura científica.

\section{GENERACIÓN MITOCONDRIAL DE RADICALES LIBRES, LONGEVIDAD Y ENFERMEDADES NO CONTAGIOSAS}

Precisando la relación entre el estrés oxidativo, el envejecimiento y las patologías propias de la edad avanzada, la información científica señala que habría dos factores involucrados. Uno de ellos y que corresponde al primero en ser identificado, relaciona la velocidad de envejecimiento con la velocidad de generación de especies reactivas de oxígeno o radicales libres a nivel mitocondrial. Los estudios realizados han señalado que la producción de ROS de las mitocondrias aisladas de tejidos post mitóticos es menor en las especies longevas que en las especies de vida corta, esto ocurre en casi todos los animales homeotermos longevos, independiente de su tasa de consumo de oxígeno (14). Este importante hallazgo explicaría, porqué la correlación entre antioxidantes y longevidad máxima es negativa: el nivel de antioxidantes en los animales longevos es bajo por el simple hecho de que la intensidad de producción de ROS es menor (13). Sin embargo, la evidencia demuestra que una mayor concentración tisular de antioxidantes, si bien no afecta la longevidad, si tiene efecto sobre la esperanza de vida gracias a su efecto preventivo en la aparición y/o progresión de enfermedades no contagiosas como la diabetes (30).

El segundo factor que permite efectuar la conexión entre el estrés oxidativo y la velocidad de envejecimiento, corresponde al hallazgo de que el daño oxidativo al ADN mitocondrial de estructuras como el corazón, hígado y cerebro correlaciona en forma negativa con la longevidad máxima en animales homeotermos, que incluye aves y mamíferos, correlación inexistente en el caso del ADN nuclear, situación que puede incrementarse con la edad (15). La acumulación del daño en el ADN mitocondrial que en el ratón se da entre los 2 y 3 años, requiere en el Chimpancé de 35 a 50 años y en el hombre de un tiempo estimado entre 70 y 100 años (13).

Con respecto a las enfermedades no contagiosas, la evidencia experimental permite un amplio nivel de aceptación respecto a que la sobre producción de especies reactivas de oxigeno (ROS) o la deficiencia en las defensas intracelulares contra los ROS (figura $N^{\circ} 1$ ), se traducirá en la patogénesis

\section{Estrés oxidativo}

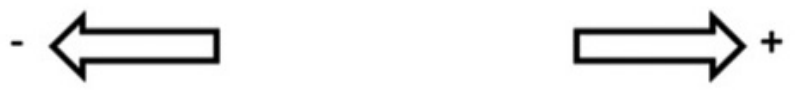

Exógenos: (vitaminas C, E, flavonoides), otros

Endógenos (Super oxido dismutasa, Glutatión peroxidasa, otros).

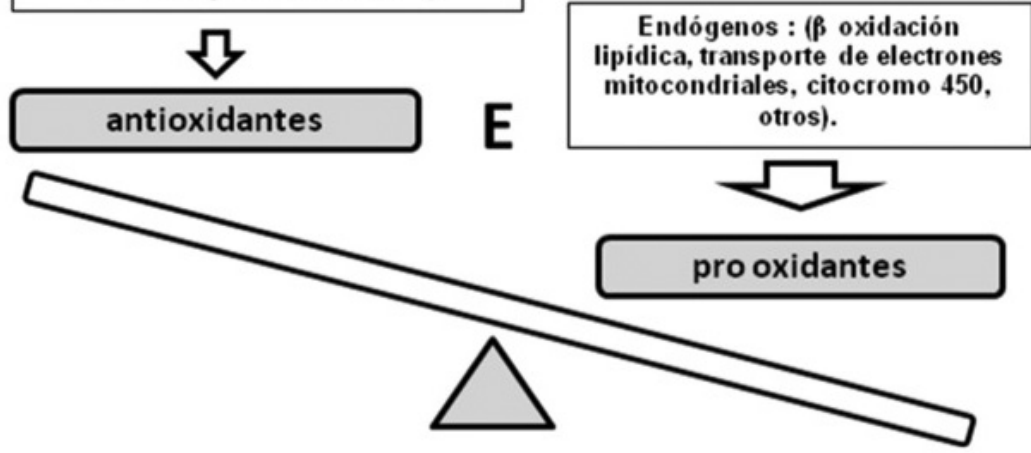

Exógenos : (grasas, tabaco, radiación), otros.

Endógenos : $(\boldsymbol{\beta}$ oxidación lipidica, transporte de electrones otros). 
de alteraciones de salud como la diabetes tipo $2(16,30)$. El daño causado por ROS implica además la presencia de procesos inflamatorios relacionados con la formación de tumores. Reaven y col, señalaron que al menos el $25 \%$ de los individuos no diabéticos presentan resistencia a la insulina, el deterioro en la tolerancia a la glucosa se produce cuando aumenta la resistencia a la insulina o las respuestas secretoras de compensación de insulina disminuyen. El aumento de insulina y/o glucosa y de ácidos grasos libres favorece la producción de ROS y el estrés oxidativo, que puede empeorar tanto la acción como la secreción de insulina acelerando la progresión a diabetes tipo $2(17,27)$.

El efecto de la RC en la producción mitocondrial de ROS se ha investigado en tejidos de rata, mostrando una significativa disminución en la tasa de generación de radicales libres en las mitocondrias de cerebro, corazón e hígado (23). La figura 1 muestra como el estrés oxidativo es el descontrol de la oxidación, tanto de origen endógeno como exógeno, consecuencia del desbalance de los mecanismos antioxidantes a nivel celular, El resultado será la aceleración del envejecimiento (E).

\section{HIPÓTESIS DE LA MACROAUTOFAGIA}

La macroautofagia es un proceso universal y dinámico que tiene lugar en todas las células eucariotas y comprende una reorganización de membranas subcelulares para aislar el citoplasma y los organelos dañados y transportarlos al lisosoma o vacuola, donde se degradan y reciclan (18). Este proceso es importante porque permite mantener un ajustado equilibrio entre anabolismo y catabolismo cuya finalidad es favorecer un crecimiento y desarrollo celular normal, regulado por condiciones extracelulares como la concentración de nutrientes y hormonas (19).

La macroautofagia se activa durante el ayuno cuando descienden los valores de aminoácidos e insulina (figura 2) facilitando la producción de nutrientes a partir de fuentes endógenas. Por el contrario, dentro del margen fisiológico de la concentración de aminoácidos en plasma, los valores altos (posprandiales) de insulina suprimen totalmente la macroautofagia (20). De acuerdo con esta línea del estudio de la fisiología molecular, se plantea la siguiente hipótesis: la ingesta alimentaria continua ad libitum, debiera tener como consecuencia un bloqueo, también sostenido, de la macroautofagia, lo que conlleva a una acumulación de mitocondrias, peroxisomas y componentes de membrana alterados. Del mismo modo, la RC, que por definición ofrece períodos de ayuno, prevendría o retardaría la acumulación de estos deshechos y sus consecuencias (21).

Los últimos avances en biología molecular demuestran que los genes de autofagia son fundamentales para el desarrollo y prolongación de la vida de Caenorhabditis elegans (29). En consecuencia, la autofagia se encuentra bajo el control de la nutrición y del estrés celular. En los individuos de sociedades occidentales desarrolladas cuyo acceso fácil y permanente a los alimentos han restringido el ayuno, se favorece un envejecimiento acelerado y la aparición de las enfermedades asociadas a la edad. La figura 2 muestra como estímulos externos - ayuno por ejemplo - produce inhibición de mTor induciendo el proceso de autofagia con la formación de autofagosomas (están bien establecidos los genes que intervienen en la levadura, no así en los humanos).

La macroautofagia desempeña un rol central en el envejecimiento y en el efecto de prolongación de la vida por la vía de la RC, como del mismo modo, en la muerte celular y en las enfermedades asociadas con la edad, incluyendo enfermedades neurodegenerativas y algunas formas de cáncer (21). Estudios recientes han demostrado el papel de la autofagia tanto en la promoción como en la prevención de enfermedades humanas $(18,24-26)$.

\section{ESTRÉS OXIDATIVO E INSATURACIÓN DE MEMBRANAS}

Es importante destacar como la composición lipídica de la membrana celular contribuye al estrés oxidativo. La presencia de ácidos grasos insaturados constituyen macromoléculas de gran susceptibilidad a las lesiones oxidativas, siendo tanto más expuestas a la oxidación, mientras más dobles enlaces presenten. Los estudios han mostrado que las membranas tanto celulares como mitocondriales de órganos como el hígado, corazón, músculo esquelético y riñón de mamíferos

FIGURA 2

Autofagia inducida por ayuno

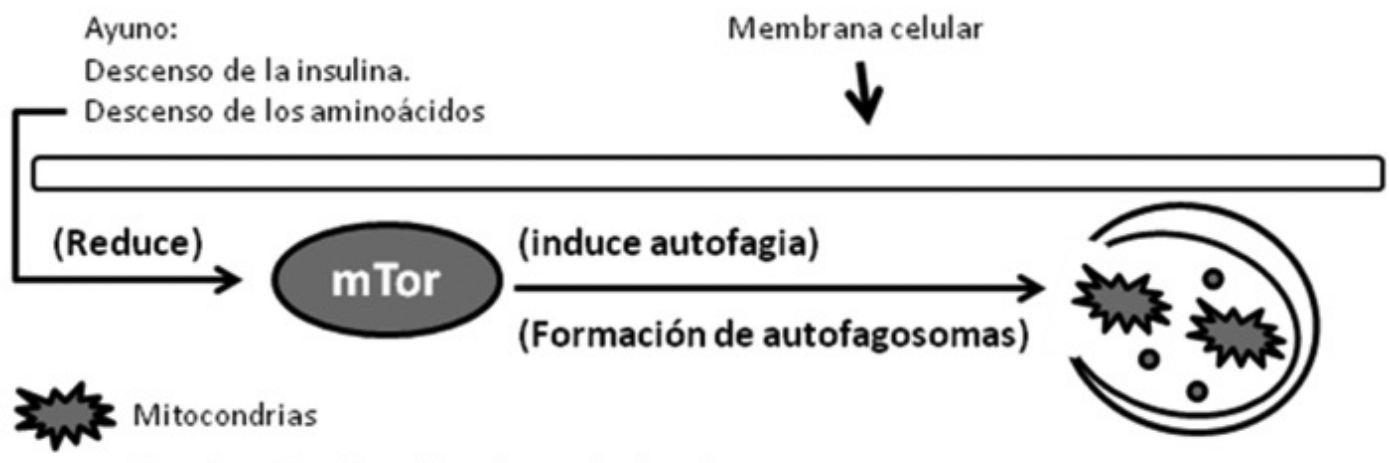

- Proteínas citosólicas/productos de desecho 
y aves longevos contienen ácidos grasos con un índice global de insaturación más bajo que los no longevos, lo que constitutivamente protege sus membranas celulares, proteínas y ADN contra la lesión derivada de peroxidación lipídica (22).

Las lesiones oxidativas, producto de la insaturación de ácidos grasos de membranas, han sido observadas también en estudios de intervención nutricional "in vivo". Dietas diseñadas para mejorar la resistencia a la peroxidación lipídica, que permitan superar la capacidad homeostática de mantenimiento de la composición de las membranas celulares de los tejidos, han permitido determinar que aceites con bajo número de dobles enlaces incorporados a la dieta de ratas, favorecen significativos cambios: induce una reducción del grado de insaturación de los ácidos grasos de membranas celulares de cerebro e hígado, disminuyen modificaciones proteicas a consecuencia de la lipoxidación, y reducen la cantidad de marcadores de daño de ADN mitocondrial $(23,31)$.

Una mayor susceptibilidad a la peroxidación lipídica de membranas muestran los sujetos con sobrepeso y obesidad, una gran cantidad de evidencia vincula una mayor susceptibilidad a la peroxidación de membranas (de eritrocitos por ejemplo) de individuos con sobrepeso y obesidad situación que se asocia con una mayor rigidez de membrana. Se ha observado una significativa correlación entre la fluidez de la membrana y la resistencia a la insulina, tanto en individuos diabéticos no dependientes de insulina, como en obesos (28).

\section{RESTRICCIÓN CALÓRICA Y DIABETES TIPO 2}

En la revisión de Hollosky y Fontana (12), los autores concluyen en que la información disponible sugiere que la restricción calórica reduce el riesgo de desarrollar diabetes tipo 2 y aterosclerosis, e induce a un número similar de respuestas de adaptación que se producen en animales de laboratorio con restricción calórica. Aunque la investigación de los efectos de la RC en los seres humanos se encuentra en una fase muy temprana, se han obtenido interesantes resultados en estudios con primates, observándose los mismos efectos antienvejecimiento que en roedores, entre otros, una menor propensión a presentar diabetes tipo 2 (7).

\section{APLICACIÓN DE LA RC EN HUMANOS}

La clásica reducción del $40 \%$ de las calorías en los trabajos de RC, es una realidad poco probable para la mayoría de los seres humanos, aunque el ofrecimiento sea el obtener beneficios de salud y mayor longevidad. Los científicos están investigando los beneficios de salud de los regimenes de RC menos estrictos y más asequibles para los seres humanos (32). Los trabajos desarrollados en este sentido sugieren que el ayuno intermitente en lugar de la restricción calórica puede ser un factor importante en la desaceleración de la tasa intrínseca de envejecimiento junto con experimentar una mejor salud (33).

\section{MODELOS DIETÉTICOS CONDUCENTES A RC}

Las principales características de lo que actualmente se considera una dieta saludable están bien establecidas (36). Se ha demostrado reiteradamente y de forma consistente que las dietas que mejor se adaptan a estos objetivos son aquellas que se basan principalmente en el consumo de frutas, verduras, hortalizas, cereales y leguminosas, utilizando con moderación los alimentos de origen animal. La dieta mediterránea se caracteriza por: alto consumo de alimentos vegetales como legumbres, cereales, frutas y verduras, frutos secos y semillas; bajo consumo de carne y productos lácteos; alto nivel de aceite de oliva y aceites vegetales en lugar de los lípidos de origen animal y el consumo moderado de vino (37). Investigadores han subrayado el papel beneficioso de este patrón dietético en el metabolismo lipídico, niveles de presión arterial e Índice de Masa Corporal, también en los procesos de inflamación y coagulación (38).

Tanto una dieta vegana baja en grasas como una dieta vegetariana basada en las guías de la ADA (Asociación Americana de Diabetes) mejoraron la glicemia y el control lipídico en pacientes diabéticos tipo 2 , beneficios que fueron mayores con la dieta vegana baja en grasas, ambas con una restricción calórica de entre 500 y 1000 calorías, 46\% de los individuos diabéticos asignados a una dieta vegana redujo los medicamentos para la diabetes tipo $2(34,35)$. Debe mencionarse que las dietas veganas han mostrado tener bajo aporte en algunos micronutrientes como retinol, vitaminas $B 12$ y $D$, calcio y zinc, del mismo modo, la evidencia muestra que micronutrientes como B1, ácido fólico, vitamina $C$, vitamina $E$, magnesio y hierro, muestran ingestas superiores a las dietas no vegetarianas $(41,42)$. Estos hallazgos vinculan a las dietas vegetarianas estrictas con algunas patologías por déficit.

La dieta DASH (enfoques alimenticios para detener la hipertensión), nace como una iniciativa del Instituto Nacional del Corazón, los Pulmones y la Sangre (NHLBI sigla en inglés), para estudiar los factores dietéticos que afectan la presión arterial. La dieta DASH tiene muchas similitudes con la dieta mediterránea. Se caracteriza por ser rica en frutas y verduras, alimentos lácticos desnatados, con reducción de la grasa total, especialmente saturada, contener cereales, papas, legumbres, frutas secas, aceite de oliva, yogurt, pescado y pollo, escasas carnes rojas y queso, vino en cantidades pequeñas. Esta dieta ha demostrado disminuir la mortalidad cardiovascular. Aunque ningún componente aislado puede explicar todos los beneficios, parece que estarían en relación a su elevado contenido en ácidos omega 3 provenientes del pescado (39).

\section{IMPLICANCIAS CLÍNICAS}

La diabetes tipo 2 constituye una de las patologías de mayor riesgo cardiovascular y por consecuencia de alta morbimortalidad (40) encuentra entre sus causas los dramáticos cambios que la sociedad ha experimentado durante los últimos 50 años, tanto en la dieta como en la actividad física.

El modelo de RC ha demostrado en animales de experimentación sus meritorios efectos sobre la longevidad y las enfermedades no trasmisibles. Si bien, en general las pautas de alimentación empleadas para el tratamiento de la diabetes, tanto como para la obesidad, se centran en la restricción de las calorías, no está claro que cumplan con el precepto de "undernutrition without mal nutrition" (reducción de la ingesta de calorías sin detrimento de la nutrición), fundamental para la preservación de la salud. Tampoco se ha indagado en la importancia de los espacios inter comidas (ayunos) los que de acuerdo con los estudios inducirían la macroautofagia la que pareciera ejercer un rol en la prevención de enfermedades no trasmisibles (ENT) y en la preservación de la salud.

Otro elemento de los estudios relacionados con la RC y longevidad, que resultan interesantes de considerar, se relaciona con los efectos que el estrés oxidativo, característico de las ENT como la diabetes, ejerce sobre la fluidez de las membranas y su efecto sobre la sensibilidad a la insulina; cambios en la composición de los aceites de la dieta podrían alterar la estructura lipídica de la membranas celulares. Estudios que consideren estos 3 aspectos: restricción calórica con equilibrio de nutrientes, programación de ayunos y cambios en la composición de los ácidos grasos de la dieta, en modelos 
con características mediterráneas, vegetarianas o tipo DASH, son necesarios para definir una pauta dietética que permita prevenir y tratar la diabetes con éxito.

\section{RESUMEN}

La restricción calórica es la única intervención nutricional que ha demostrado en forma sólida beneficios para la salud y la longevidad en animales de experimentación. Entre los efectos positivos, aquellos relacionados con la homeostasis de la glucosa son llamativos y consistentes. Pareciera que estos beneficios se vinculan con la menor generación mitocondrial de especies reactivas de oxígeno, con una menor peroxidación lipídica de la membrana celular y con la estimulación de la macroautofagia. Conocidos modelos alimenticios como dieta mediterránea, dieta vegana, vegetariana, la dieta de la población adulta de Okinawa y el modelo DASH (Enfoques Alimenticios para Detener la Hipertensión), con especial resguardo por el contenido de aceites con bajo índice de insaturación y de un estricto respeto por los ayunos inter comidas, pudieran marcar una interesante línea de construcción de un modelo dietético orientado a la prevención y tratamiento de la diabetes tipo 2 .

Palabras clave: Restricción calórica; diabetes; modelos dietéticos; tratamiento; prevención.

\section{BIBLIOGRAFÍA}

1. Weindruch, R. Caloric restriction: life span extension and retardation of brain aging. Clin Neurosc Res 2003; 2 (56): 279-84.

2. Walford, R; Liu, L; Gerbase-Delima, M; Smith, G. Longterm dietary restriction and inmmune function in mice: Response to sheep red blood cells and to mitogenic agens. Mechanisms Ageing Develop 1973; 2: 447-54.

3. Weindruch, R; Walford, RL; Fligiel, S y Guthrie, D. The retardation of aging in mice by dietary restriction: longevity, cancer, immunity and lifetime energy intake. J Nutr 1986; 116 (4): 641-54.

4. Tannenbaum, $A$. The dependence of tumor formation on the composition of the calorie - restricted diet as well as on the degree of restriction. Cancer Res 1945; 5: 616-25.

5. Pugh, $T ;$ Klopp, $R$ y Weindruch, R. Controlling caloric consumption: protocols for rodents and rhesus monkeys. Neurobiol Aging 1999; 20, 157-65.

6. Hursting, S; Lavigne, J; Berrigan, D; Perkins, Barrett1, J. Calorie restriction, Aging and Cancer Prevention: Mechanisms of Action and Applicability to Humans. Ann Rev Med 2003; 54, 131-52.

7. Roth, G; Ingram, D; Lane, M. Caloric Restriction in Primates and Relevance to Humans. Ann New York Academy Sci 2001; 928, 305-15.

8. Sohal, $R ;$ Weindruch, $R$. Oxidative Stress, Caloric Restriction, and Aging Sci 1996; 273, (5271), 59-63.

9. Kristal, B, Yu, B. An emerging hypothesis: Synergistic induction of aging by free radicals and Maillard reactions. $J$ Gerontol Biol Sci 1992; 47, B107-B14.

10. Tannenbaum, $A$. The initiaton and growth of tumor. Effects of underfeeding. Am J Cancer 1940; 38:335-59.

11. Beckman, K; Ames, Bruce. The Free Radical Theory of Aging Matures (1998). Physiol Rev 1998; 78, (2): 547-81.

12. Holloszy, J; Fontana, N. Caloric restriction in humans. Experimental Gerontol 2007; 42: 709-12.

13. Barja, G. Radicales libres de origen mitocondrial y longevidad. An Real Academia Nacional Farmacia 2005; 71: 783-98.

14. Pérez-Campo, R; López - Torres, M; Cadenas, S; Rojas, C y Barja, G: The rate of free radical production as a determinant of the rate of aging: evidence from the comparative approach. J Comparative Physiol B 1998, 168: 149-58.

15. Barja, G; Herrero, A. Oxidative damage to mitochondrial DNA is inversely related to maximum life span in the heart and brain of mammals. FASEB J 2000, 14: 312-8.

16. Newsholme, P; Haber, E; Hirabara, S; Rebelato, E; Procopio, J; Morgan, D; Oliveira-Emilio, H; A. R. Carpinelli y Curi, R. Diabetes associated cell stress and dysfunction: role of mitochondrial and non-mitochondrial ROS production and activity. J Physiol 2007, 583.1: 9-24.

17. Evans, J; Goldfine, I; Maddux, B; Grodsky, G. Are Oxidative Stress_Activated Signaling Pathways Mediators of Insulin Resistance and Cell Dysfunction?. Diabetes 2003, 52 (1):1-8.

18. Klionsky, D; Emr, S. Autophagy as a Regulated Pathway of Cellular Degradation. Science 2000, 290 (5497): 1717-21.

19. Bergamini, E. El mecanismo de prolongación de la vida por restricción calórica y la intensificación farmacológica de sus efectos. Rev Española Geriatría Gerontol 2005; 40 (2), 108-13.

20. Mortimore, G; Poso, R. Intracellular protein catabolism and its control during nutrient deprivation and supply. Ann Rev Nutr 1987; 7:539-64.

21. Couture $P$, Hulbert $A$. Membrane fatty acid composition of tissues is related to body mass of mammals. J Membrane Biol 1995; 148 (1): 27-39.

22. Barja, G. Free radicals and aging. TRENS Neurosci 2004; 27 (10): 595-600.

23. Shintani, T; Klionsky, D. Autophagy in Health and Disease: A Double-Edged Sword. Science 2004; 306: 990-5.

24. Ellington, $A$; Berhow, $M$; Singletary, $K$ : Induction of macroautophagy in human colon cancer cells by soybean B-group triterpenoid saponins. Carcinogenesis 2005; 26 (1): 159-67.

25. Eskelinen, E; Saftig, P. Autophagy: A lysosomal degradation pathway with a central role in health and disease. Biochim Biophys Acta 1793, 2009; 664-73.

26. Stephens, J; Khanolkar, M y Bain, S. The biological relevance and measurement of plasma markers of oxidative stress in diabetes and cardiovascular disease. Atherosclerosis 2009, 202: 321-9.

27. Cazzola, R; Rondanelli; M; Russo-Volpe, S; Ferrari, E y Benvenutto, C. Decreased membrane fluidity and altered susceptibility to peroxidation and lipid composition in overweight and obese female erythrocytes. J Lipid Res 2004; 45: 1846-51.

28. Kang, C; You, Y; Avery, L. Dual roles of autophagy in the survival of Caenorhabditis elegans during starvation. Genes Develop 2007, 21: 2161-71.

29. Maritim, $A$; Sanders, $R$ y Watkins, J. Diabetes, Oxidative Stress, and Antioxidants: A Review. J Biochem Molecular Toxicol 2000, 17 (1): 24-37.

30. Pamplona, R; Barja, G y Portero - Otín, M. Membrane Fatty Acid Unsaturation, Protection against Oxidative Stress, and Maximum Life Span. Ann New York Academy of Sci 2002, 959: 475-90.

31. Dirks, A y Leeuwenburgh, C. Caloric restriction in humans: Potential pitfalls and health concerns. Mechanisms Ageing and Develop 2006, 127: 1-7.

32. Anson, R; Guo, Z; de Cabo, R; Lyun, T; Rios, M; Hagepanos, A; Ingram, D; Lane, $M$ y Mattson, $M$. Intermittent fasting dissociates beneficial effects of dietary restriction on glucose metabolism and neuronal resistance to injury from 
calorie intake. Proc National Academy Sci (PNAS) 2003, 100 (10): 6216-20.

33. Barnard, N; Cohen, J; Jenkins, D; Turner-Mc Grievy, G; Gloede, L; Jaster, B; Deidl, K; Green, A y Talpers, S.A Low-Fat Vegan Diet Improves Glycemic Control and Cardiovascular Risk Factors in a Randomized Clinical Trial in Individuals With Type 2 Diabetes. Diabetes Care 2006, 29 (8): 1777-83.

34. Turner-McGrievy, G; Barnard, N; Cohen, J; Jenkins, D; Gloede, L y Green, A Changes in Nutrient Intake and Dietary Quality among Participants with Type 2 Diabetes Following a Low-Fat Vegan Diet or a Conventional Diabetes Diet for 22 Weeks. J Am Diet Assoc 2008, 108 (10): 1636-45.

35. Carbajal, A y Ortega, R. La Dieta Mediterránea como Modelo de Dieta Prudente y Saludable. Rev Chil Nutr 2001, 28 (2): 224-36.

36. Garine, I. Dieta mediterránea y Estilo de Vida. VIII Congreso
Internacional de Barcelona sobre la Dieta Mediterránea, Barcelona 24 y 25 de marzo 2010.

37. Lorgeril, M y Salem, P. The Mediterranean-style diet for the prevention of cardiovascular diseases. Public Health Nutr 2006; 9 (1A): 118-23.

38. Sosa, J. "Tratamiento no farmacológico de la hipertensión arterial". An Facultad Med 2010, 71 (4): 241-4.

39. Bennett, C; Guo, M y Dharmage, S. HbA1c as a screening tool for detection of Type 2 diabetes: a systematic review. Diabetic Med 2007; 24: 333-43.

40. Davey, G; Spencer, E; Appleby, P; Allen, N; Knox, Ky Key, T. EPIC-Oxford: lifestyle characteristics and nutrient intakes in a cohort of 33883 meat-eaters and 31546 non meateaters in the UK. Public Health Nutr 2003; 6: 259-68.

41. Waldmann, A; Koschizke, JW; Leitzmann, C y Hahn, A.Dietary intakes and lifestyle factors of a veganpopulation in Germany: results from the GermanVegan Study. European J Clin Nutr 2003; 57: 947-55. 